\title{
PHYTOCHEMICAL ANALYSIS OF SELECTED INDIAN MEDICINAL PLANTS BY HR-LCMS SPECTRA METHOD
}

\author{
Nateshan Anil ${ }^{1,2}$ and Venkateswara Rao Talluri ${ }^{3, 凶}$ \\ ${ }^{1}$ Department of Biotechnology, KL Deemed to be University, Vaddeswaram-522 502, \\ Andhra Pradesh, India. \\ ${ }^{2}$ Department of Biotechnology, Sreenidhi Institute of Science and Technology, \\ Hyderabad-500056, Telangana, India. \\ ${ }^{3}$ Prof. TNA Innovation Center, Varsha Biosciences and Technology India Private Limited, \\ Sy.No.253/A, Jiblakpally(V), Dothigudem(GP), Boodhan Pochampally(M), Yadadri District- \\ 508284, Telangana, India \\ Corresponding Author: vrtalluri@gmail.com
}

\begin{abstract}
Due to the widespread use of medicinal plant products in the pharmaceutical and biotechnology field, phytochemical analysis of medicinal plants has become an important and challenging task. Analytical techniques including liquid chromatography coupled with mass spectrometry were found to be an important technique in the analysis of complex bioactive constituents. The present study was aimed at bioactive constituent analysis from select Indian medicinal plants viz., Pongamia pinnata, Dodonea viscosa, Gardenia resinifera and Gymnospora emarginata by (HR)-LCMS (High Resolution- Liquid Chromatography Mass Spectrometry) analysis. The present study confirms presence of important therapeutic secondary metabolites including alkaloids (Hydroxycotinine), Flavonoids (Apiin, Genkwanin, Kaempherol), Coumarin (Lomatin), Carboxylic acid (Quinic acid) and Phenolic compounds (Isoliquiritigenin) in the select Indian medicinal plants.
\end{abstract}

Keywords: HR-LCMS, Anthelmentic Activity, Antibacterial Activity, Phytochemicals.

RASĀYAN J. Chem., Vol. 14, No.4, 2021

\section{INTRODUCTION}

The role of bioactive compounds extracted from medicinal plants in maintaining sustainable human health globally is well documented. The traditional medicinal practices including Rigveda (3700 B.C.), Ayurveda, Homeopathy and Unani mentioned the use of medicinal plant products for the cure of various human ailments. ${ }^{1,2}$ In the recent times, many widely used drugs with low side effects were sourced from medicinal plants. There is a great demand for the identification of novel, potent drug molecules from medicinal plant products that are safe with low side effects to treat infections caused by parasites and microbial pathogens. ${ }^{3,4}$

In the biochemical analysis of plant products, the first step is the identification and isolation of bioactive compounds from the medicinal plants. In the present study, based on the literature survey, four Indian medicinal plants viz., Pongamia pinnata, Dodonea viscosa, Gardenia resinifera, Gymnospora emarginata were selected from Ananthagiri forest of Telangana ${ }^{5}$ and their extracts were analyzed using (HR)-LCMS (High-resolution liquid chromatography-mass spectrometry) for the identification of bioactive compounds. Pongamia pinnata also known as karum tree belongs to the family Fabaceae. All the parts of this plant are shown to exhibit various medicinal properties. People suffering from bleeding piles are treated with Pongamia pinnata bark extract and fruits are used as antidyslipidemic. Oil extracted from seeds is used in the treatment of rheumatism, leucoderma and scabies. The extract of seeds and leaves is shown to have anthelmintic and insecticidal activities. ${ }^{6,7}$

Dodonea viscosa commonly called hop bush belongs to the family Sapindaceae. This plant is widely used globally for treating various ailments. Leaf and bark extract shows antibacterial activity ${ }^{8}$, antidiabetic activity in rats, ${ }^{9}$ anticancer activity against lung and breast cancer cell lines. It is also found to be effective in treating rheumatism, malaria, and snake bites. ${ }^{10}$

Rasayan J. Chem., 14(4), 2318-2326(2021)

http://doi.org/10.31788/RJC.2021.1446473

This work is licensed under a CC BY 4.0 license. 
Gardenia resinifera belongs to the family Rubiaceae is commonly called Indian boxwood. The various plant parts of it shown different medicinal properties and among them, gum extract from stems is found to show antihyperlipidemic activity and hepatoprotective effects. The leaf extract is used in treating pathogenic bacteria of the mouth. Methanolic extract of leaves is found to be anthelmintic and antispasmodic. ${ }^{11,12}$

Gymnospora emarginata, commonly called Danthi belongs to the family Celastraceae. The leaf extract is found to be effective in controlling various gram positive and gram negative bacteria ${ }^{13}$, in treating different cancers in humans (Hep3b hepatocellular carcinoma, hela; cervical epithelial cancer and A549 human lung adrenal cancer $)^{14}$ along with antioxidant activity. ${ }^{15}$

\section{Collection of Medicinal Plants}

\section{EXPERIMENTAL}

The leaves of Dodonea viscosa, Gymnospora emarginata, Gardenia resinifera and seeds of Pongamia pinnata were collected from Ananthagiri forest Vikarabad District Telangana, India. The plant specimens were submitted to Botany Department, Osmania University, Hyderabad and Botany Department, Kakatiya University, Warangal for the taxonomic identification of plants. One of the plant specimens was identified as Dodonea viscosa (L) Jacq (family Sapindaceae, Voucher number 0073) and deposited in the Department of Botany, Osmania University, Hyderabad. The other three plant specimens were identified as Gymnospora emarginata (family Celastraceae, Voucher number KUW 4529), Gardenia resinifera (family Rubiaceae, Voucher number KUW 4528) and Pongamia pinnata (family Leguminosae, Voucher number KUW4557) and deposited in the Department of Botany, Kakatiya University, Warangal.

\section{Extraction of Bioactive Compounds}

The leaves and seeds of select medicinal plants were finely powdered and bioactive compounds were extracted with methanol using a Soxhlet extractor. ${ }^{16}$ The methanol extracts of Dodonea viscosa, Gymnospora emarginata, Gardenia resinifera and Pongamia pinnata were filtered through Whatman filter paper no 42 individually and concentrated using an evaporator at $40^{\circ} \mathrm{C}$ and stored at $4{ }^{\circ} \mathrm{C}$.

\section{High Resolution -Liquid Chromatography Mass Spectrometry(HR-LCMS) Methodology}

The methanolic extracts of Pongamia pinnata, Dodonea viscosa, Gardenia resinifera, Gymnospora emarginata were subjected to (HR)-LCMS analysis individually and chemical fingerprints were prepared using high-resolution liquid chromatography and mass spectrometry (model-G6550A of Agilent technologies) with $0.01 \%$ mass resolution ${ }^{17}$ with following parameters:

1. MS- minimum range $150(\mathrm{M} / \mathrm{Z})$ and maximum 1000 daltons with scanning rate each per Second.

2. The source parameter for gas chromatography was maintained at $250{ }^{\circ} \mathrm{C}$ with a gas flow of 13 psi/minute.

3. The auxiliary draw speed was $100 \mu \mathrm{l} /$ minute, eject speed at $100.0 \mu \mathrm{L} / \mathrm{min}$, draw position offset 0.0 $\mathrm{mm}$ wait time after drawing $2.0 \mathrm{~s}$, Sample flush out factor was 5.0.

The following solvent composition was used:

\begin{tabular}{c|c|c|c|c|c|c|c}
\hline S.No. & Channel & Ch.1 Solvent & Name 1 & Ch2 Solvent & Selected & Used & Percent \\
\hline 1 & A & $100.0 \%$ & $0.1 \%$ FA in water & $100.0 \%$ Water V.02 & Ch.2 & yes & $95.00 \%$ \\
& & Water V.02 & & & & & \\
\hline 2 & B & $100.0 \%$ & $90 \%$ ACN $+10 \%$ & $100.0 \%$ & Ch.2 & yes & $5.00 \%$ \\
& & AcetonitrileV.02 & H2O+ 0.1\% FA & Acetonitrile & & & \\
\hline
\end{tabular}

\section{RESULTS AND DISCUSSION}

The high resolution-liquid chromatography-mass spectrometry analysis (HR)-LCMS of methanolic extract of Pongamia pinnata seeds was found to contain 52 compounds of which, 35 major compounds were confirmed based on their retention time, mass, and molecular formula as shown in Table-1, chromatogram, Figure-1. The chromatogram gives information on the relative concentrations of various compounds eluted as a function of retention time. 
RASĀYAN J. Chem.

Vol. 14 | No. 4 |2318-2326| October- December | 2021

The height of the peak indicates the relative concentrations of bioactive compounds. Mass Spectrometer analyses the structure of unknown compounds which are eluted at different times.

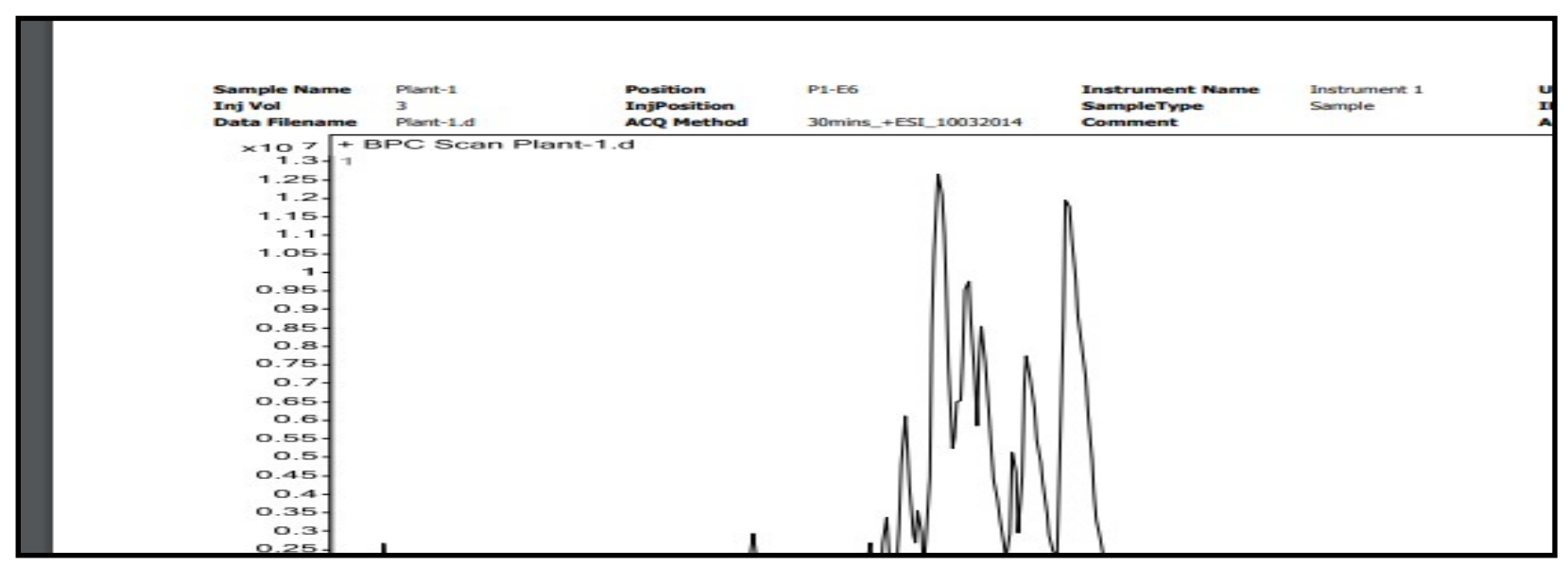

Fig-1 :( HR)-LCMS Spectrogram of Pongamia pinnata

The important phytoconstituents confirmed by (HR)-LCMS Analysis were Apiin, Juglone, PHydroxyphenylacetic acid, Betaxolol, Artelinic acid, Picrotoxinin, Dihydrodeoxystreptomycin, Isoliquiritigenin, Sterigmatocystin, Hydroxyflutamide, Formononetin, Genkwanin, Dicumarol, Vulpinic acid, Lomatin, Chryophanic acid, 9-anthrone Isoliquiritigenin, Dihydrosphingosine. The compounds were also found in other plants which have shown different pharmacological activities. Among them, Juglone a 1-4 naphtha quinone compound found in Juglans nigra plant has shown potent antibacterial activity against various species. ${ }^{18,19}$ Vulpinic acid which was also found in lichens has shown anticancer activity and antibacterial activity. ${ }^{20,21}$ Cucurbitacins have shown anticancer, antidiabetic, anti-inflammatory effects. $^{22}$

Table-1: Bioactive Compounds identified in Methanol Extract of Pongamia pinnata

\begin{tabular}{c|l|l|c|c|c}
\hline S. No. & \multicolumn{1}{|c|}{ Name of the Compound } & Formula & Mass & $\begin{array}{c}\text { RT } \\
(\mathrm{min})\end{array}$ & $\begin{array}{c}\text { DBDiff } \\
(\mathrm{ppm})\end{array}$ \\
\hline 1. & $\begin{array}{l}\text { Mebeverine metabolite (veratric acid } \\
\text { glucuronide) }\end{array}$ & $\mathrm{C}_{15} \mathrm{H}_{18} \mathrm{O}_{10}$ & 358.0855 & 1.013 & 12.46 \\
\hline 2. & 12 a-hydroxy-5- deoxydehydromunduser one & $\mathrm{C}_{19} \mathrm{H}_{18} \mathrm{O}_{6}$ & 342.1122 & 1.018 & -5.5 \\
\hline 3. & 6,3 -dimethoxyflavone & $\mathrm{C}_{17} \mathrm{H}_{14} \mathrm{O}_{4}$ & 282.0843 & 4.669 & 17.36 \\
\hline 4. & $\begin{array}{l}\text { galnacalpha(1-3)[fucalpha(1- 2)]galbeta(1- } \\
\text { 4)glcnacbeta- sp }\end{array}$ & $\mathrm{C}_{30} \mathrm{H}_{51} \mathrm{~N}_{5} \mathrm{O}_{20}$ & 801.3232 & 5.102 & -13.06 \\
\hline 5. & Apiin & $\mathrm{C}_{26} \mathrm{H}_{28} \mathrm{O}_{14}$ & 564.1434 & 5.786 & 7.92 \\
\hline 6. & beta-erythroidine & $\mathrm{C}_{16} \mathrm{H}_{19} \mathrm{~N} \mathrm{O}_{3}$ & 273.1365 & 6.911 & -0.01 \\
\hline 7. & Juglone & $\mathrm{C}_{10} \mathrm{H}_{6} \mathrm{O}_{3}$ & 174.0306 & 7.218 & 6.3 \\
\hline 8. & p-hydroxyphenylacetic acid & $\mathrm{C}_{8} \mathrm{H}_{8} \mathrm{O}_{3}$ & 152.048 & 7.949 & -4.49 \\
\hline 9. & betaxolol & $\mathrm{C}_{18} \mathrm{H}_{29} \mathrm{~N} \mathrm{O}_{3}$ & 307.2123 & 8.134 & 8 \\
\hline 10. & 3a-oh desogestrel & $\mathrm{C}_{22} \mathrm{H}_{30} \mathrm{O}_{2}$ & 326.2289 & 8.322 & -13.35 \\
\hline 11. & s-adenosylmethionine & $\mathrm{C}_{15} \mathrm{H}_{23} \mathrm{~N}_{6} \mathrm{O}_{5} \mathrm{~S}$ & 399.1452 & 8.433 & -0.46 \\
\hline 12. & Artelinic acid & $\mathrm{C}_{23} \mathrm{H}_{30} \mathrm{O}_{7}$ & 418.1967 & 8.86 & 5.76 \\
\hline 13. & Picrotoxinin & $\mathrm{C}_{15} \mathrm{H}_{16} \mathrm{O}_{6}$ & 292.0948 & 8.861 & -0.42 \\
\hline 14. & Dihydrodeoxystreptomycin & $\mathrm{C}_{21} \mathrm{H}_{41} \mathrm{~N}_{7} \mathrm{O}_{11}$ & 567.2856 & 9.305 & 1.48 \\
\hline 15. & trans-3-hydroxycotinine glucuronide & $\mathrm{C}_{16} \mathrm{H}_{20} \mathrm{~N}_{2} \mathrm{O}_{8}$ & 368.1233 & 9.697 & -3.74 \\
\hline 16. & Sappanone a 7-methyl ether & $\mathrm{C}_{17} \mathrm{H}_{14} \mathrm{O}_{5}$ & 298.0818 & 9.755 & 7.8 \\
\hline 17. & Isoliquiritigenin & $\mathrm{C}_{15} \mathrm{H}_{12} \mathrm{O}_{4}$ & 256.0741 & 10.145 & -2.17 \\
\hline 18. & Sterigmatocystin & $\mathrm{C}_{18} \mathrm{H}_{12} \mathrm{O}_{6}$ & 324.0612 & 10.635 & 6.77 \\
\hline 19. & estradiol-17beta 3-sulfate & $\mathrm{C}_{18} \mathrm{H}_{24} \mathrm{O}_{5} \mathrm{~S}$ & 352.129 & 10.726 & 15.46 \\
\hline 20. & 12a-hydroxy-5- deoxydehydromunduser one & $\mathrm{C}_{19} \mathrm{H}_{18} \mathrm{O}_{6}$ & 342.1081 & 10.798 & 6.41 \\
\hline 21. & Deoxysappanone b trimethyl ether & $\mathrm{C}_{19} \mathrm{H}_{20} \mathrm{O}_{5}$ & 328.1277 & 10.831 & 10.37 \\
\hline
\end{tabular}


RASĀYAN J. Chem.

Vol. 14 | No. 4 |2318-2326| October- December | 2021

\begin{tabular}{|c|c|c|c|c|c|}
\hline 22. & Hydroxyflutamide & $\begin{array}{l}\mathrm{C}_{11} \mathrm{H}_{11} \mathrm{~F}_{3} \\
\mathrm{~N}_{2} \mathrm{O}_{4}\end{array}$ & 292.0718 & 11.044 & -16.02 \\
\hline 23. & deoxysappanone $b$ trimethyl ether & $\mathrm{C}_{19} \mathrm{H}_{20} \mathrm{O}_{5}$ & 328.1313 & 11.204 & -0.54 \\
\hline 24. & 8,13-dihydroxy-9,11-octadecadienoic acid & $\mathrm{C}_{18} \mathrm{H}_{32} \mathrm{O}_{4}$ & 312.2279 & 11.23 & 7.03 \\
\hline 25. & Formononetin & $\mathrm{C}_{16} \mathrm{H}_{12} \mathrm{O}_{4}$ & 268.0741 & 11.24 & -1.98 \\
\hline 26. & n,n-Didemethylchlorpromazine & $\mathrm{C}_{15} \mathrm{H}_{15} \mathrm{C}_{1} \mathrm{~N}_{2} \mathrm{~S}$ & 290.0594 & 11.299 & 17.38 \\
\hline 27. & Cuneatin methyl ether & $\mathrm{C}_{18} \mathrm{H}_{14} \mathrm{O}_{6}$ & 326.0771 & 11.456 & 5.92 \\
\hline 28. & Sterigmatocystin & $\mathrm{C}_{18} \mathrm{H}_{12} \mathrm{O}_{6}$ & 324.0615 & 11.462 & 5.96 \\
\hline 29. & Genkwanin & $\mathrm{C}_{16} \mathrm{H}_{12} \mathrm{O}_{5}$ & 284.0693 & 11.613 & -3.07 \\
\hline 30. & 5-o-methylvisamminol & $\mathrm{C}_{16} \mathrm{H}_{18} \mathrm{O}_{5}$ & 290.1153 & 11.647 & 0.51 \\
\hline 31. & 6,3'-dimethoxyflavone & $\mathrm{C}_{17} \mathrm{H}_{14} \mathrm{O}_{4}$ & 282.0877 & 11.675 & 5.48 \\
\hline 32. & Chrysophanic acid 9- anthrone & $\mathrm{C}_{15} \mathrm{H}_{12} \mathrm{O}_{3}$ & 240.0799 & 11.736 & -5.15 \\
\hline 33. & Dalbergione, 4-methoxy- 4'-hydroxy- & $\mathrm{C}_{16} \mathrm{H}_{14} \mathrm{O}_{4}$ & 270.0902 & 11.783 & -3.64 \\
\hline 34. & Juglone & $\mathrm{C}_{10} \mathrm{H}_{6} \mathrm{O}_{3}$ & 174.0307 & 11.949 & 5.66 \\
\hline 35. & 4-hydroxyphenylethanol & $\mathrm{C}_{8} \mathrm{H}_{10} \mathrm{O}_{2}$ & 138.0699 & 11.956 & -13.09 \\
\hline 36. & Warfarin & $\mathrm{C}_{19} \mathrm{H}_{16} \mathrm{O}_{4}$ & 308.1033 & 11.957 & 5.01 \\
\hline 37. & i-methyl-4-nitro-5-thio- imidazole & $\mathrm{C}_{4} \mathrm{H}_{5} \mathrm{~N}_{3} \mathrm{O}_{2} \mathrm{~S}$ & 159.0082 & 11.963 & 13.17 \\
\hline 38. & Dicumarol & $\mathrm{C}_{19} \mathrm{H}_{12} \mathrm{O}_{6}$ & 336.0618 & 11.966 & 4.83 \\
\hline 39. & Hydroxyflutamide & $\mathrm{C}_{11} \mathrm{H}_{11} \mathrm{~F}_{3} \mathrm{~N}_{2} \mathrm{O}_{4}$ & 292.0723 & 12.27 & -17.74 \\
\hline 40. & Vulpinic acid & $\mathrm{C}_{19} \mathrm{H}_{14} \mathrm{O}_{5}$ & 322.0826 & 12.378 & 4.78 \\
\hline 41. & Lomatin & $\mathrm{C}_{14} \mathrm{H}_{14} \mathrm{O}_{4}$ & 246.088 & 13.113 & 4.79 \\
\hline 42. & Methyl robustone & $\mathrm{C}_{22} \mathrm{H}_{18} \mathrm{O}_{6}$ & 378.1087 & 13.513 & 4.21 \\
\hline 43. & 3'-hydroxy-e,e-dienoestrol & $\mathrm{C}_{18} \mathrm{H}_{18} \mathrm{O}_{3}$ & 282.1262 & 13.525 & -2.17 \\
\hline 44. & Propargite & $\mathrm{C}_{19} \mathrm{H}_{26} \mathrm{O}_{4} \mathrm{~S}$ & 350.1497 & 13.608 & 15.66 \\
\hline 45. & 5,7-dimethoxyisoflavone & $\mathrm{C}_{17} \mathrm{H}_{14} \mathrm{O}_{4}$ & 282.0911 & 13.862 & -6.78 \\
\hline 46. & $\begin{array}{l}\text { 3,5-Pyridinedicarboxylic acid,1,4-dihydro-2,6- } \\
\text { dimethyl-4-(3nitrophenyl)-, monomethyl ester }\end{array}$ & $\mathrm{C}_{16} \mathrm{H}_{16} \mathrm{~N}_{2} \mathrm{O}_{6}$ & 332.1032 & 13.906 & -7.09 \\
\hline 47. & Benzylbutylphthalate & $\mathrm{C}_{19} \mathrm{H}_{20} \mathrm{O}_{4}$ & 312.137 & 13.929 & -2.59 \\
\hline 48. & Isoliquiritigenin & $\mathrm{C}_{15} \mathrm{H}_{12} \mathrm{O}_{4}$ & 256.0746 & 13.944 & -4 \\
\hline 49. & 5c-aglycone & $\mathrm{C}_{16} \mathrm{H}_{16} \mathrm{O}_{4}$ & 272.1056 & 14.318 & -2.64 \\
\hline 50. & Juglone & $\mathrm{C}_{10} \mathrm{H}_{6} \mathrm{O}_{3}$ & 174.0306 & 14.341 & 6.01 \\
\hline 51. & Avocadene acetate & $\mathrm{C}_{19} \mathrm{H}_{36} \mathrm{O}_{4}$ & 328.2591 & 14.364 & 6.83 \\
\hline 52. & Lomatin & $\mathrm{C}_{14} \mathrm{H}_{14} \mathrm{O}_{4}$ & 246.0878 & 14.875 & 5.66 \\
\hline
\end{tabular}

The (HR)-LCMS High-Resolution Liquid Chromatography Mass Spectrometer analysis of methanolic extract of Dodonea viscosa spectrum profile (Fig.-2) shows 33 compounds of which 29 major compounds were confirmed based on their retention time, mass and molecular formula.

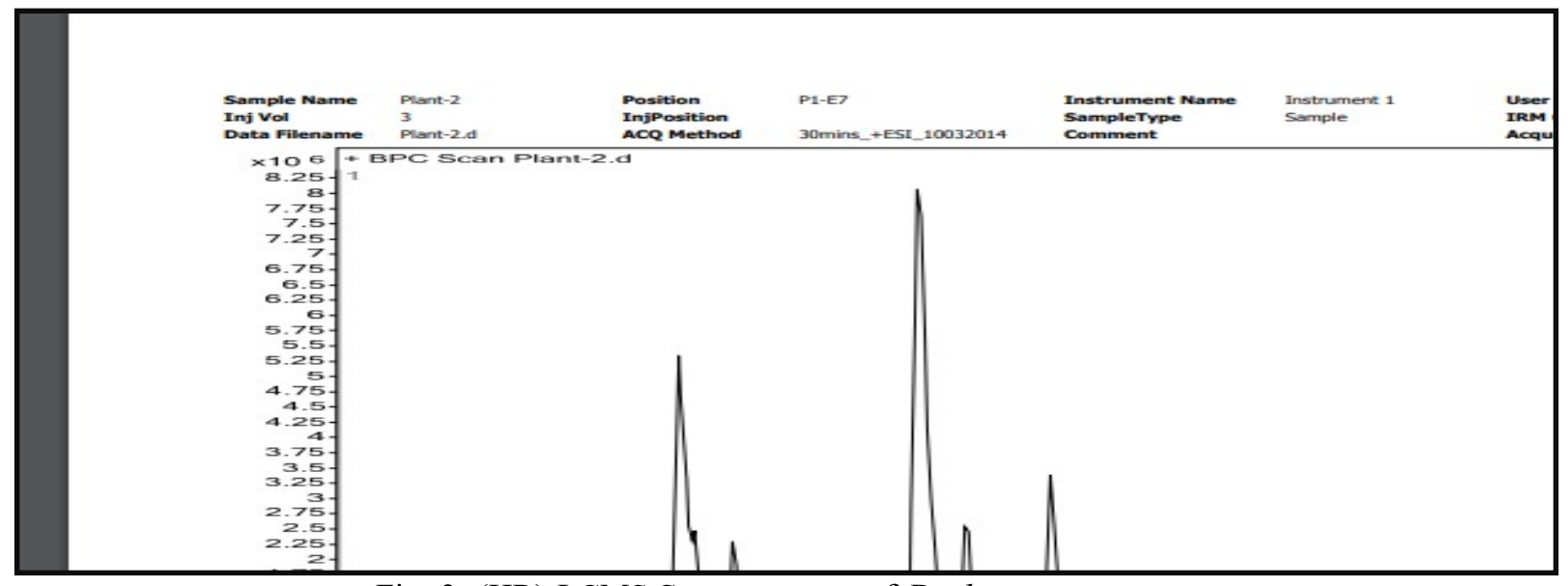

Fig -2: (HR)-LCMS Spectrogram of Dodonea viscosa 
RASĀYAN J. Chem.

Vol. 14 | No. 4 |2318-2326| October- December | 2021

The Phytochemicals found in the extract including Fraxetin, Hieracin, Desmethylzopiclone, Kaempherol, Tamarixetin, Epothilone A, 4Hydroxyestrone, 4'Hydroxypenbutololglucuronide, Orthothymotinic acid, Dihydrodeoxystreptomycin, Piretanide, Rutin, Irbesartan, Eupatorin were shown in Table-2.

It was also reported that these compounds found in the different species of plants exhibit different pharmacological activities. Among them, Kaempherol was also found in tea, berries and apples exhibits antitumor activity and anti-inflammatory activity.

Fraxetin also found in Fraxinus rhynchophylla has shown antibacterial activity and antioxidant effect. Rutin also found in tea leaves and apples exhibited various pharmacological effects like antidiabetic, antiinflammatory, antibacterial activity. ${ }^{23-25}$

Table -2: Bioactive Compounds in Methanol Extract of Dodonea viscosa

\begin{tabular}{|c|c|c|c|c|c|}
\hline S. No. & Name of the Compound & Formula & Mass & $\begin{array}{c}\mathrm{RT} \\
(\mathrm{min})\end{array}$ & $\begin{array}{c}\text { DBDiff } \\
(\mathrm{ppm})\end{array}$ \\
\hline 1. & $\begin{array}{l}\text { Mebeverine metabolite (Veratric acid } \\
\text { glucuronide) }\end{array}$ & $\mathrm{C}_{15} \mathrm{H}_{18} \mathrm{O}_{10}$ & 358.0848 & 1.035 & \\
\hline 2. & $\begin{array}{l}\text { 12a-Hydroxy-5- } \\
\text { Deoxydehydromunduser one }\end{array}$ & $\mathrm{C}_{19} \mathrm{H}_{18} \mathrm{O}_{6}$ & 342.1112 & 1.06 & 14.62 \\
\hline 3. & Fraxetin & $\mathrm{C}_{10} \mathrm{H}_{8} \mathrm{O}_{5}$ & 208.0347 & 5.21 & -2.49 \\
\hline 4. & Hieracin & $\mathrm{C}_{15} \mathrm{H}_{10} \mathrm{O}_{7}$ & 302.0389 & 6.281 & \\
\hline 5. & Desmethylzopiclone & $\mathrm{C}_{16} \mathrm{H}_{15} \mathrm{C}_{1} \mathrm{~N}_{6} \mathrm{O}_{3}$ & 374.0807 & 6.446 & 12.35 \\
\hline 6. & Kaempherol & $\mathrm{C}_{15} \mathrm{H}_{10} \mathrm{O}_{6}$ & 286.0446 & 6.546 & 23.31 \\
\hline 7. & Tamarixetin & $\mathrm{C}_{16} \mathrm{H}_{12} \mathrm{O}_{7}$ & 316.0547 & 6.678 & 11.12 \\
\hline 8. & Epothilone A & $\mathrm{C}_{26} \mathrm{H}_{39} \mathrm{~N} \mathrm{O}_{6} \mathrm{~S}$ & 493.2499 & 6.78 & 11.36 \\
\hline 9. & $\begin{array}{l}\text { 1-Cyclohexene-1-acrylic acid, 2,6,6- } \\
\text { trimethyl-3-oxo- }\end{array}$ & $\mathrm{C}_{12} \mathrm{H}_{16} \mathrm{O}_{3}$ & 208.1099 & 7.707 & \\
\hline 10. & 4-Hydroxyestrone & $\mathrm{C}_{18} \mathrm{H}_{22} \mathrm{O}_{3}$ & 286.1539 & 7.709 & 0.43 \\
\hline 11. & 4'-Hydroxypenbutolol glucuronide & $\mathrm{C}_{24} \mathrm{H}_{37} \mathrm{~N} \mathrm{O}_{9}$ & 483.2426 & 7.71 & 10.3 \\
\hline 12. & 6,8,10,12-pentadecatetraenal & $\mathrm{C}_{15} \mathrm{H}_{22} \mathrm{O}$ & 218.167 & 7.71 & 8.74 \\
\hline 13. & Orthothymotinic acid & $\mathrm{C}_{11} \mathrm{H}_{14} \mathrm{O}_{3}$ & 194.0945 & 7.713 & 0.32 \\
\hline 14. & 17alpha-Estradiol 3-D- glucuronide & $\mathrm{C}_{24} \mathrm{H}_{32} \mathrm{O}_{8}$ & 448.2033 & 8.024 & 3.34 \\
\hline 15. & 4-Hydroxyestrone & $\mathrm{C}_{18} \mathrm{H}_{22} \mathrm{O}_{3}$ & 286.1573 & 8.433 & 14.35 \\
\hline 16. & $\begin{array}{l}\text { 2,4,6-Heptatrienoic acid, 5- methyl-7- } \\
\text { (2,6,6-trimethyl-3- oxo-1-cyclohexen- } \\
\text { 1-yl)-, (2E,4E,6E)- }\end{array}$ & $\mathrm{C}_{17} \mathrm{H}_{22} \mathrm{O}_{3}$ & 274.1568 & 8.438 & -1.45 \\
\hline 17. & $\begin{array}{l}\text { 2-Methylene-5-(2,5- } \\
\text { Dioxotetrahydrofuran- 3-yl)-6-oxo-- } \\
\text { 10,10- Dimethylbicyclo[7: 2: } \\
\text { 0]Undecane }\end{array}$ & $\mathrm{C}_{18} \mathrm{H}_{24} \mathrm{O}_{4}$ & 304.1665 & 8.446 & 0.19 \\
\hline 18. & thyrotropin releasing hormone & $\mathrm{C}_{16} \mathrm{H}_{22} \mathrm{~N}_{6} \mathrm{O}_{4}$ & 362.1699 & 8.455 & 3.26 \\
\hline 19. & 7-hydroxy Tetranor Iloprost & $\mathrm{C}_{18} \mathrm{H}_{26} \mathrm{O}_{5}$ & 322.1779 & 8.463 & 0.9 \\
\hline 20. & $\begin{array}{l}\text { 17beta-Estradiol 3-(beta-D- } \\
\text { glucuronide) }\end{array}$ & $\mathrm{C}_{24} \mathrm{H}_{32} \mathrm{O}_{8}$ & 448.2029 & 8.515 & 0.47 \\
\hline 21. & 16b-Hydroxyestradiol & $\mathrm{C}_{18} \mathrm{H}_{24} \mathrm{O}_{3}$ & 288.172 & 8.54 & 15.13 \\
\hline 22. & Gibberellin A44 diacid & $\mathrm{C}_{20} \mathrm{H}_{28} \mathrm{O}_{6}$ & 364.1851 & 8.558 & 1.8 \\
\hline 23. & 2,9-heptadecadien-4,6-diyn- 1,8-diol & $\mathrm{C}_{17} \mathrm{H}_{24} \mathrm{O}_{2}$ & 260.1769 & 8.604 & 9.65 \\
\hline 24. & $\begin{array}{l}\text { 5-hydroperoxy-7-[3,5- epidioxy-2-(2- } \\
\text { octenyl)- cyclopentyl]-6-heptenoic acid }\end{array}$ & $\mathrm{C}_{19} \mathrm{H}_{30} \mathrm{O}_{6}$ & 354.2031 & 8.781 & 2.66 \\
\hline 25. & 4-Hydroxyestrone & $\mathrm{C}_{18} \mathrm{H}_{22} \mathrm{O}_{3}$ & 286.1545 & 9.123 & -2.5 \\
\hline 26. & Dihydrodeoxystreptomycin & $\mathrm{C}_{21} \mathrm{H}_{41} \mathrm{~N}_{7} \mathrm{O}_{11}$ & 567.2848 & 9.31 & \\
\hline 27. & Gibberellin A15 & $\mathrm{C}_{20} \mathrm{H}_{28} \mathrm{O}_{5}$ & 348.1908 & 9.444 & 2.8 \\
\hline 28. & Piretanide & $\mathrm{C}_{17} \mathrm{H}_{18} \mathrm{~N}_{2} \mathrm{O}_{5} \mathrm{~S}$ & 362.0975 & 9.857 & 8.12 \\
\hline 29. & Alprazolam & $\mathrm{C}_{17} \mathrm{H}_{13} \mathrm{Cl} \mathrm{N} \mathrm{N}_{4}$ & 308.0897 & 9.877 & -10.73 \\
\hline 30. & Rutin & $\mathrm{C}_{27} \mathrm{H}_{30} \mathrm{O}_{16}$ & 610.151 & 6.495 & \\
\hline 31. & Irbesartan & $\mathrm{C}_{25} \mathrm{H}_{28} \mathrm{~N}_{6} \mathrm{O}$ & 428.2403 & 8.661 & -5.08 \\
\hline
\end{tabular}


RASĀYAN J. Chem.

Vol. 14 | No. 4 |2318-2326| October- December | 2021

\begin{tabular}{c|l|l|c|c|c}
\hline 32. & Gibberellin A44 diacid & $\mathrm{C}_{20} \mathrm{H}_{28} \mathrm{O}_{6}$ & 364.1887 & 9.034 & 1.53 \\
\hline 33. & 18-Hydroxycortisol & $\mathrm{C}_{21} \mathrm{H}_{30} \mathrm{O}$ & 378.2038 & 10.933 & 3.24 \\
\hline
\end{tabular}

The (HR)-LCMS High-Resolution Liquid Chromatography-Mass Spectrometer analysis of Gardenia resinifera methanolic extract spectrum profile Fig.-3 shows 43 compounds of which 27 major compounds were confirmed based on their retention time, mass, and molecular formula.

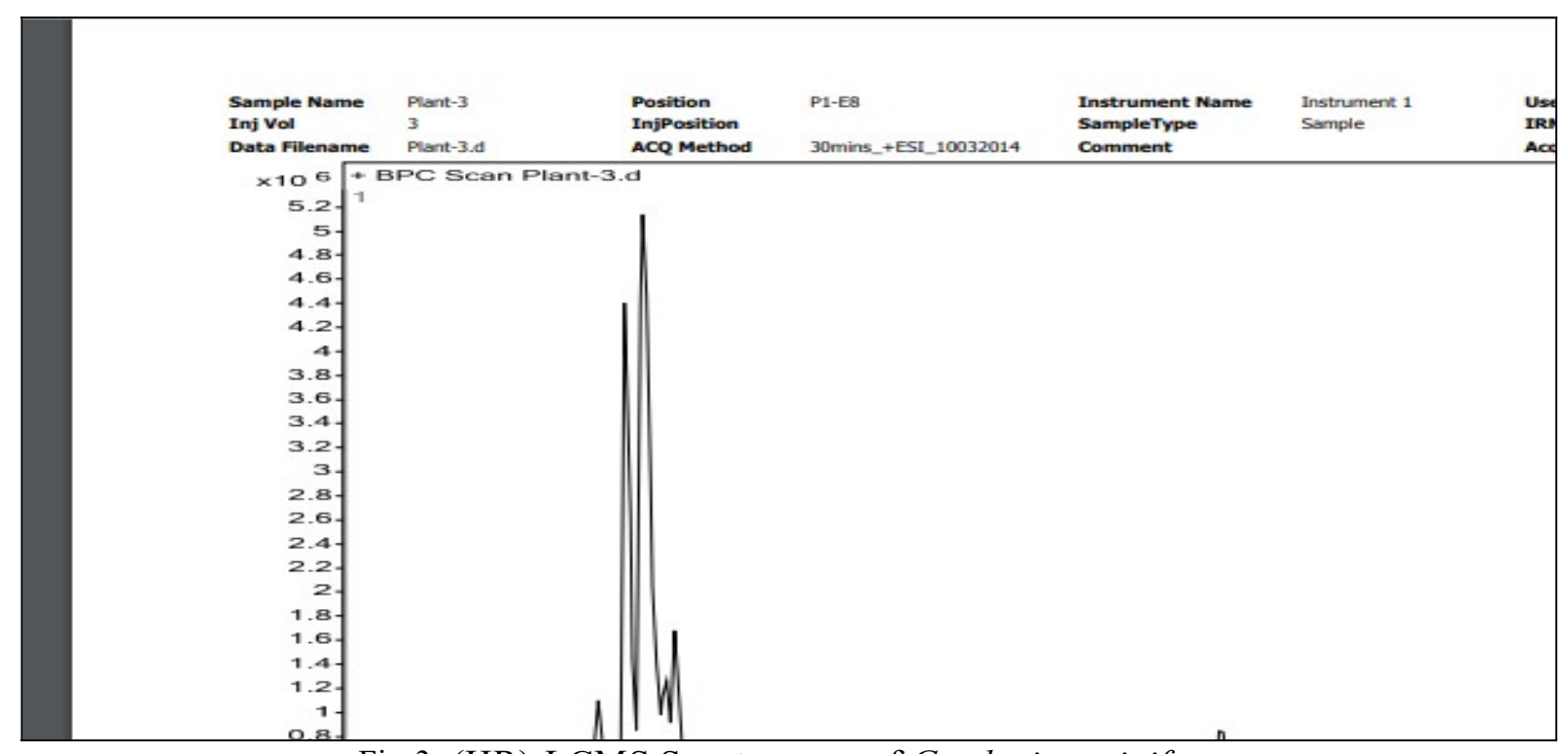

Fig-3: (HR)-LCMS Spectrogram of Gardenia resinifera

The phytochemicals identified in the extract includes Chlorogenic acid, Ecgonine, Loganin, Kaempherol, Hieracin, Warfarin, Benzylbutylphthalate, Promazine sulfoxide, Quinic acid, Hyroxyhydroquinone, Khayanthone and Bergenin were shown in table -3. Various authors reported different pharmacological activities for these compounds. Among them, Loganin found in Strychnos nux-vomica L shows central nervous stimulant activity and anti-inflammatory activity ${ }^{27}$. Quinic acid obtained from flowers of Moringa olifera exhibited various pharmacological activities including anticancer activity and antibacterial activity ${ }^{28,29}$ Genkwanin found in Callicarpa Americana revealed various pharmacological activities including anti-inflammatory, antibacterial and anticancer properties. ${ }^{30}$

The High-Resolution Liquid Chromatography-Mass Spectrometer (HR-LCMS) analysis of Gymnospora emarginata methanolic extract spectrum profile Fig.-4 shows 19 compounds of which 15 major compounds were confirmed based on their retention time, mass, and molecular formula.

Table-3: Bioactive Compounds in Methanol Extract of Garedenia resinifera

\begin{tabular}{c|l|l|c|c|c}
\hline S. No. & \multicolumn{1}{|c|}{ Name } & Formula & Mass & RT & $\begin{array}{c}\text { DB Diff } \\
\text { (ppm) }\end{array}$ \\
\hline 1. & $\begin{array}{l}\text { Mebeverine metabolite (Veratric acid } \\
\text { glucuronide) }\end{array}$ & $\mathrm{C}_{15} \mathrm{H}_{18} \mathrm{O}_{10}$ & 358.0856 & 1.042 & 12.29 \\
\hline $\begin{array}{c}3,5-\text {-Pyridinedicarboxylic acid, 2,6-dimethyl- } \\
\text { 4-(3- nitrophenyl)-, mono(2- hydroxyethyl) } \\
\text { ester }\end{array}$ & $\mathrm{C}_{17} \mathrm{H}_{16} \mathrm{~N}_{2} \mathrm{O}_{7}$ & 360.0993 & 1.043 & -9.86 \\
\hline 3. & Deoxyelephantopin & $\mathrm{C}_{19} \mathrm{H}_{20} \mathrm{O}_{6}$ & 344.1244 & 1.045 & 4.53 \\
\hline 4. & 12a-Hydroxy-5- Deoxydehydromunduser one & $\mathrm{C}_{19} \mathrm{H}_{18} \mathrm{O}_{6}$ & 342.1115 & 1.05 & -3.28 \\
\hline 5. & Bis (2-hydroxypropyl) amine & $\mathrm{C}_{6} \mathrm{H}_{15} \mathrm{~N} \mathrm{O}_{2}$ & 133.1103 & 1.14 & -0.39 \\
\hline 6. & 4-Hydroxy-L-threonine & $\mathrm{C}_{4} \mathrm{H}_{9} \mathrm{~N} \mathrm{O}_{4}$ & 135.0524 & 1.363 & 5.47 \\
\hline 7. & $\begin{array}{l}\text { cis-3-(6-Hydroxy-7-methoxy- 5- } \\
\text { benzofuranyl)acrylic acid glucuronide }\end{array}$ & $\mathrm{C}_{18} \mathrm{H}_{18} \mathrm{O}_{11}$ & 410.0763 & 2.862 & 20.95 \\
\hline 8. & 4-Amino-m-cresol & $\mathrm{C}_{7} \mathrm{H}_{9} \mathrm{~N} \mathrm{O}$ & 123.0686 & 4.228 & -1.41 \\
\hline
\end{tabular}


RASĀYAN J. Chem.

Vol. 14 | No. 4 |2318-2326| October- December | 2021

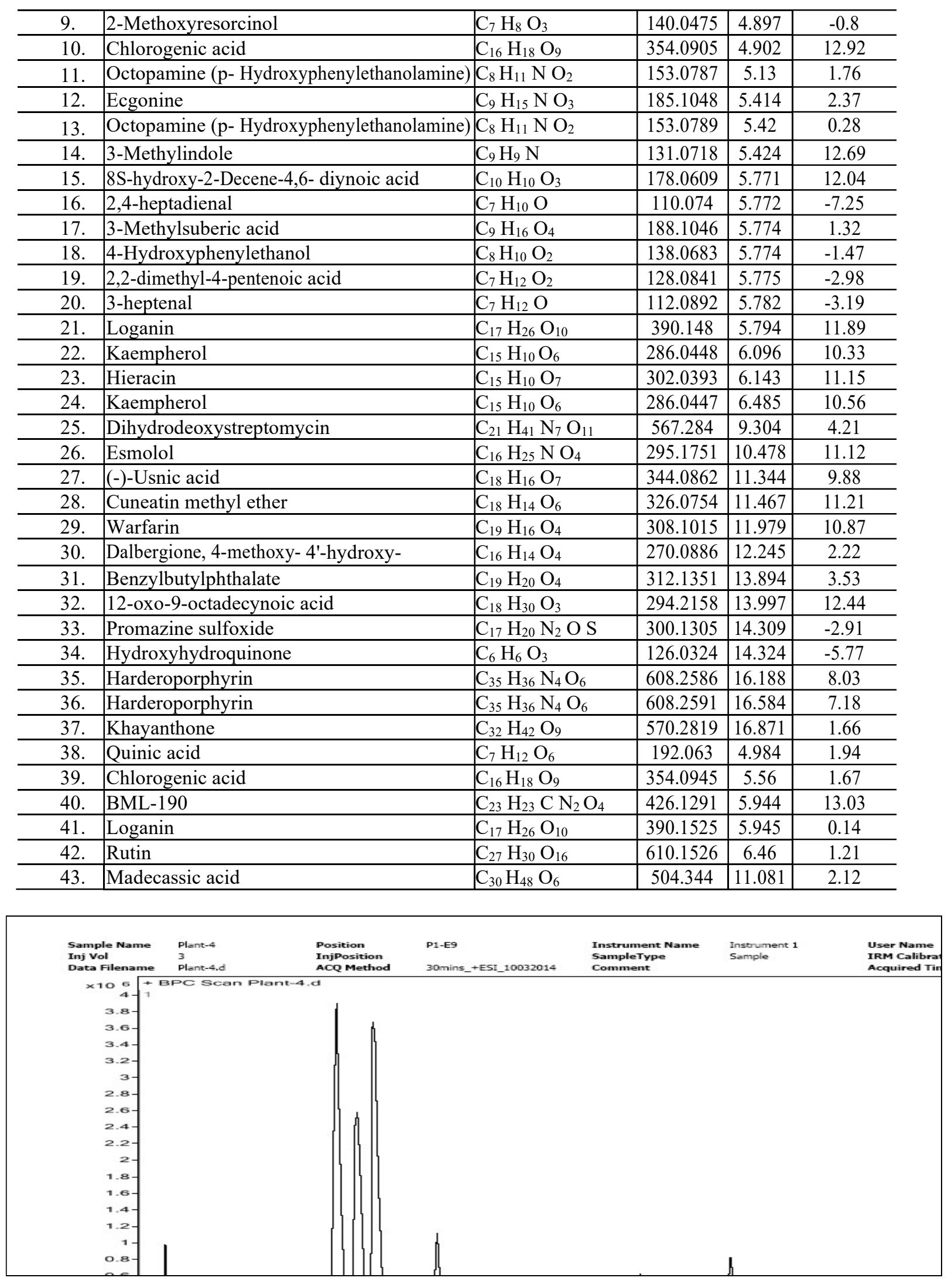

Fig- 4: (HR)-LCMS Spectrogram of Gymnospora emarginata 
RASĀYAN J. Chem.

Vol. 14 | No. 4 |2318-2326| October- December | 2021

The phytochemicals found in the extract are Proline, Thiabendazole, Disulfiram, Tamarixetin, Luteoline, Pargyline, Oxdemetonmethyl, Naphazoline, Dihydrodeoxystreomptycin (Table-4). These compounds were also shown to be present in other plants and exhibit antimicrobial, antioxidant, anticancer, antiinflammatory activity. ${ }^{31}$

Table-4: Bioactive Compounds in Methanol Extract of Gymnospora emarginata

\begin{tabular}{r|l|l|c|c|c}
\hline S. No. & Name & Formula & Mass & Rt & DB Diff (ppm) \\
\hline 1. & 2a-hydroxy-5- deoxydehydromunduser one & $\mathrm{C}_{19} \mathrm{H}_{18} \mathrm{O}_{6}$ & 42.1115 & 1.047 & -3.28 \\
\hline 2. & Thiabendazole & $\mathrm{C}_{10} \mathrm{H}_{7} \mathrm{~N}_{3} \mathrm{~S}$ & 201.0333 & 4.728 & 13.87 \\
\hline 3. & Disulfiram & $\mathrm{C}_{10} \mathrm{H}_{20} \mathrm{~N}_{2} \mathrm{~S}_{4}$ & 296.0518 & 5.921 & -2.89 \\
\hline 4. & Luteoline & $\mathrm{C}_{15} \mathrm{H}_{10} \mathrm{O}_{6}$ & 286.0441 & 6.114 & 12.7 \\
\hline 5. & Pargyline & $\mathrm{C}_{11} \mathrm{H}_{13} \mathrm{~N}$ & 159.1026 & 6.195 & 14.1 \\
\hline 6. & Tamarixetin & $\mathrm{C}_{16} \mathrm{H}_{12} \mathrm{O}_{7}$ & 316.0541 & 6.198 & 13.25 \\
\hline 7. & Luteoline & $\mathrm{C}_{15} \mathrm{H}_{10} \mathrm{O}_{6}$ & 286.0443 & 6.645 & 12.13 \\
\hline 8. & Naphazoline & $\mathrm{C}_{14} \mathrm{H}_{14} \mathrm{~N}_{2}$ & 210.115 & 8.706 & 3.51 \\
\hline 9. & Dihydrodeoxystreptomycin & $\mathrm{C}_{21} \mathrm{H}_{41} \mathrm{~N}_{7} \mathrm{O}_{11}$ & 567.2839 & 9.323 & 4.44 \\
\hline 10. & Uroporphyrinogen III & $\mathrm{C}_{40} \mathrm{H}_{44} \mathrm{~N}_{4} \mathrm{O}_{16}$ & 836.2714 & 12.105 & 4.54 \\
\hline 11. & Promazine sulfoxide & $\mathrm{C}_{17} \mathrm{H}_{20} \mathrm{~N}_{2} \mathrm{O} \mathrm{S}$ & 300.1301 & 14.293 & -1.49 \\
\hline 12. & Hydroxyhydroquinone & $\mathrm{C}_{6} \mathrm{H}_{6} \mathrm{O}_{3}$ & 126.0322 & 14.306 & -4.05 \\
\hline 13. & 1 -Methylinosine & $\mathrm{C}_{11} \mathrm{H}_{14} \mathrm{~N}_{4} \mathrm{O}_{5}$ & 282.0993 & 14.699 & -10.03 \\
\hline 14. & Mometasone Furoate & $\mathrm{C}_{27} \mathrm{H}_{30} \mathrm{C}_{12} \mathrm{O}_{6}$ & 520.1406 & 15.508 & 2.5 \\
\hline 15. & Avobenzone & $\mathrm{C}_{20} \mathrm{H}_{22} \mathrm{O}_{3}$ & 310.1534 & 15.951 & 11.41 \\
\hline 16. & $\begin{array}{l}\text { 12beta-Hydroxy-3-oxo-5beta- cholan-24-oic } \\
\text { Acid }\end{array}$ & $\mathrm{C}_{24} \mathrm{H}_{38} \mathrm{O}_{4}$ & 390.2729 & 18.448 & 10.55 \\
\hline 19 & Hydroxyhydroquinone & $\mathrm{C}_{6} \mathrm{H}_{6} \mathrm{O}_{3}$ & 126.0322 & 18.489 & -4.26 \\
\hline
\end{tabular}

\section{CONCLUSION}

The Methanolic extract of Pongamia pinnata, Dodonea viscosa, Gardenia resinifera, Gymnospora emarginata revealed the presence of therapeutically important bioactive compounds like flavonoids, glycosides, alkaloids, coumarins, terpenoids, saponins using (HR)-LCMS high-resolution liquid chromatography-mass spectrometer analysis. These bioactive components possess important pharmacological activities and could be useful for treating various human ailments. Further, invitro and insilico studies were planned on these bioactive compounds to identify candidate drug molecules for treating various parasitic diseases including filariasis.

\section{ACKNOWLEDGEMENT}

The authors are immensely thankful to the Department of Botany Osmania University, Telangana Department of Botany, Kakatiya University, Telangana for the identification of plant species, Department of Biotechnology, Sreenidhi Institute of Science and Technology, Telangana for the permission to utilize the lab facilities and University Grants Commission (UGC) for the project research grant (Reference no. F.N0:4-5t4/ 2015-16(MRP / UGC-SERO).

\section{REFERENCES}

1. A. Balkrishna, R. K. Mishra, A. Srivastava, B. Joshi, R. Marde and U.B. Prajapati, International Journal of Unani and Integrative Medicine, 3, 40(2019).

2. M. M. Pandey, S. Rastogi, A. K. S. Rawat, Evidence-Based Complementary and Alternative Medicine, 6, (2013), https://doi.org/10.1155/2013/376327

3. M. Lahlou, Pharmacology \& Pharmacy, 4, 17(2013), https://doi.org/10.4236/pp.2013.43A003 
RASĀYAN J. Chem.

Vol. 14 | No. 4 |2318-2326| October- December | 2021

4. A. K. Patra, Dietary Phyochemicals and Microbes, Springer, Dordrecht, p.1,32,(2012).

5. D. S. Pawar and S. Nasreen, Journal of Medicinal Plants Studies, 6, 173, (2018).

6. V. V. Chopade, A. N. Tankar1, V. V. Pande1, A.R. Tekade1, N.M. Gowekar1, S.R. Bhandari, S. N. Khandake1, International Journal of Green Pharmacy, 4 (2008).

7. P. Sowjanya , P. Srinivasa Babu, D. N. Lakshmi, N. Navyasri, Y. Harshini, J . Vyshnavi and M. Prasanth, Journal of Pharmacognosy Phytochemistry, 7, 459 (2018),

8. R. Naidoo, M. Patel , Z. Gulube, and I. Fenyvesi , Journal of Ethnopharmacology, 144, 71174(2012), https://doi.org/10.1016/j.jep.(2012).08.045

9. M. S. Akhtar, M. Ahmed, K. Gulzar and H. Adnan, Diabetologia Croatica , 40 ,71(2011).

10. B. Anil Reddy, Journal of Pharmaceutical Science and Technology, 1,1(2009).

11. B. Jhansi Lakshmi and K. Jaganmohanreddy, Biosciences Biotech Research Communications, 4, 23(2011).

12. C.P. Khare, Indian Medicinal Plants, Springer, (2007).

13. R. Gupta, M. G. Vairale, R. R. Deshmukh, P. R. Chaudhary, Indian Journal of Traditional Knowledge, 9,713(2010).

14. S. Sagwan, D. V. Rao, R. A. Sharma, Asian Journal of Pharmaceutical and Clinical Research, 4, 3(2011).

15. B. Dhanasree, N. S. Basha, Journal of Pharmacognosy and Phytochemistry, 4,123(2015).

16. R. James, Journal of Microbiology \& Biology Education, 15, 45(2014), https://doi:10.1128/jmbe.v15i1.656

17. J. J. Pitt, Mass Spectrometry in Clinical Biochemistry Reviews, 30, 2(2009).

18. Wang Jiayi, Cheng Yuhuan, Wu Rina, Jiang Donghua, Bing Bai, Tan Dehong, Yan Tingcai, Sun Xiyun, Zhang Qi, Wu Zhaoxia, International Journal of Molecular Science, 17(6), 965 (2016), https://doi.org/10.3390/ijms17060965.

19. A. M. Clark, T. M. Jurgens, C. D. Hufford, Phytotherapy Research, 4,11(1990), https://doi.org/10.1002/ptr.2650040104

20. N. Kılıç, M. K. Derici , İ. Büyü, S. S. Aydın, A. Süme, D. C. Duman, Indian journal of Pharmaceutical Education and Research, 52, 4(2018), https://doi.org/10.5530/ijper.52.4.73

21. M. L. Wein, M. Oethinger, K. Belsner, T. Peters, and R. Marre, Antimicrobial Agents and Chemotherapy, 995, 2541, https://doi.org/10.1128/AAC.39.11.2541

22. H. C. Kee, X. Hongtao, US, 2008/0207578 A1, 28(2008).

23. C. Y. Hsu, H. Y. Shih, H. Y. Y. C. Chia, C. H. Lee, H. Ashida, Y. K. Lai , C. F. Weng, Molecular Nutrition \& Food Research ,58, 1168 (2014), https://doi.org/10.1002/mnfr.201300691

24. H. Javed, M. M. Khan, A. Ahmad, K. Vaibhav, M. E. Ahmad, A. Khan, M. Ashafaq, F. Islam, M. S. Siddiq, M. M. Safhi, F. Islam, Neuroscience, 17,340 (2012).

25. M. K. Araruna, S. A. Brito, M. F. Morais Braga, K. K. Santosh, T. M. Souza, T. R. Leite , J. G. Costa, H. D. Coutinho, Indian Journal Medical Research, 135,252(2012).

26. I. Sarfraz, A. Rasul, F. Jabeen, T. Younis, Md. Kashif, Z . Muhammad, A. Muhammad Ali, Evidence Based Complementary and Alternative Medicine, 12 (2017), https://doi.org/10.1155/2017/4269

27. M. Khan, A. Garg, S. K. Srivastava and M. P. Darokar, Medicinal Chemistry Research, 21, 2975(2012), https://doi.org/10.1007/s00044-011-9832-9

28. S. Muthamil , B.B.Balamurugan , S.K.Pandian .Front Microbiolology, 9,2835(2018) , https://doi.org/:10.3389/fmicb 2018.02835.

29. E. Padmini and L. Inbathamizh, Asian Journal of Pharmaceutical and Clinical Research, 6(4), 106(2013).

30. G. Porras, J. Bacsa, H. Tang, C. L. Quave, Crystals, 9, 491(2019), https://doi.org/10.3390/crysta19100491

31. Y. Li., R. Shi, X. Wang, H. Shen, Current Cancer Drug Targets, 8, 634(2008), https://doi.org/10.2174/156800908786241050

[RJC-6473/2021] 